\title{
Diffraction-Contrast Analysis of Dislocation Loops in BCC Alloys
}

\author{
J. Bentley
}

Metals \& Ceramics Division, Oak Ridge National Laboratory, PO Box 2008, Oak Ridge, TN 37831-6064

Soon after the first observations of dislocations by transmission electron microscopy (TEM), dislocation loops in $\mathrm{MgO}$ were analyzed by Groves and Kelly and their character determined as interstitial; some months later, after Mike Whelan pointed out the extra $180^{\circ}$ rotation between image and diffraction pattern, the loop character was changed to vacancy [1]. It can be argued that loop analysis has been in a continuing state of confusion ever since. In the first of five seminal papers on irradiation damage in molybdenum, Maher and Eyre described in detail the analysis of non-edge perfect dislocation loops, including the concept of "safe orientations" where contrast is in the same sense as that of a pure edge loop [2]. Needless to say, some confusion followed, even in print [3].

Dislocation loop analysis was an integral part of a TEM characterization study of Mo neutronirradiated at elevated temperatures to fluences of 1 and $3 \times 10^{20}$ fission neutrons.cm ${ }^{-2}$ [4]. With one exception, all clearly resolved loops were interstitial in character for materials irradiated at $\geq 475^{\circ} \mathrm{C}$. Remarkably, in TZM alloy (Mo-0.5\% Ti-0.1\%Zr) irradiated at 750 and $850^{\circ} \mathrm{C}$ high concentrations of small $(<20 \mathrm{~nm}$ diameter) vacancy loops with Burgers vector $\mathbf{b}=\mathrm{a} / 2<111>$ constituted the dominant component of the damage microstructure. The $100-\mathrm{kV}$ diffraction-contrast analyses followed a procedure derived from Maher and Eyre [2] that accounted for foil normals near $<011>$. All dislocation loops with $\mathbf{b}=\mathrm{a} / 2<111>$ were imaged with diffracting vector $\mathbf{g}= \pm 200$ at a beam direction (image-plane normal) $\mathbf{B}=$ [023]. Images recorded with $\mathbf{g}=01 \overline{1}$ at $\mathbf{B}=[155], \mathbf{g}=1 \overline{2} 1$ at $\mathbf{B}=[135]$ and $\mathbf{g}=121$ at $\mathbf{B}=[137]$ were used to identify loops with $\mathbf{b}= \pm \mathrm{a} / 2[111]$ and $\pm \mathrm{a} / 2[111]$ from $\mathbf{g} \cdot \mathbf{b}=0$ invisible or residual contrast. Both $+\mathbf{g}$ and $-\mathbf{g}$ diffracting vectors were used (with $\mathbf{s}_{\mathrm{g}}$ constant and positive) so that the invariance in strength of contrast and position of images satisfying $\mathbf{g} \cdot \mathbf{b}=0$ conditions could be confirmed. Diffracting vectors in safe orientations which gave $\mathbf{g} \cdot \mathbf{b}= \pm 2$ conditions for the appropriate loops were used for inside/outside contrast analyses to determine the sense of $\mathbf{b}$ and thus the nature of the loop. For $\mathbf{b}= \pm \mathbf{a} / 2[111], \pm \mathbf{g}=310$ at $\mathbf{B}=[\overline{136}]$ and for $\mathbf{b}= \pm \mathrm{a} / 2[\overline{1} 11]$, $\pm \mathbf{g}=\overline{3} 10$ at $\mathbf{B}=[136]$ were used. Weak-beam dark-field images were also recorded for the inside/outside $\mathbf{g . b}= \pm 2$ analyses with $\mathbf{S}_{<310>}=2.4$ to $2.9 \times 10^{-2} \AA^{-1}$ (Ewald sphere intersecting the reciprocal lattice $1 / 4$ to $1 / 2$ of the distance from $<620>$ to $<930>$ ). Outside contrast occurs for $(\mathbf{g . b}) \mathrm{s}_{\mathrm{g}}$ $>0$, inside contrast for $(\mathbf{g} \cdot \mathbf{b}) \mathrm{s}_{\mathrm{g}}<0$. With $\mathbf{b}$ defined by the FS/RH perfect crystal convention, the positive dislocation direction as clockwise when viewed from above, and $\mathbf{n}=$ upward loop normal, for interstitial loops $\mathbf{n . b}>0$ and for vacancy loops $\mathbf{n} . \mathbf{b}<0$. The presence of vacancy loops and their stability during post-irradiation annealing was rationalized on the basis of segregation of oversized $\mathrm{Ti}$ and $\mathrm{Zr}$ solutes to the dilated near-core regions, or through the formation of Ti-Zr-C complexes [4].

$\mathrm{V}-4 \% \mathrm{Cr}-4 \% \mathrm{Ti}$ has been of interest for the last decade as a candidate structural material for proposed fusion reactors. For a series of oxygen-doped alloys, annealing at $950^{\circ} \mathrm{C}$ resulted in the formation of large (diameter $>1 \mu \mathrm{m}$ ), disk-shaped, TiC-rich Ti(C,O,N) precipitates $\sim 2 \mathrm{~nm}$ thick on $\{001\}$ [5]. The misfit normal to the habit gives rise to misfit dislocation loops. Diffraction-contrast analyses with $\pm \mathbf{g}$ $=<110>$ and $<200>$ reveal that $\mathbf{b} \approx \mathbf{a}<001>$, although the exact magnitude of $\mathbf{b}$ is uncertain since displacement fringes are commonly present with $\mathbf{g}=<110>$ (figure 1). Since the loops are of pure edge type, no consideration of safe orientations is needed and inside/outside contrast analyses with $\mathbf{g . b}= \pm 2$ are achieved with $\mathbf{g}=<112>$, as shown in figure 2 . The loops have interstitial character, as expected from the bi-phase crystallography. The results are directly relevant to analysis of similar precipitates and secondary point-defect clusters in neutron-irradiated $\mathrm{V}-4 \% \mathrm{Cr}-4 \% \mathrm{Ti}$. Even with the added complications of ferromagnetism, similar analyses of interstitial loops with $\mathbf{b}=\mathbf{a}<100>$ were performed by Horton and Bentley on neutron-irradiated $\mathrm{Fe}$ and ion-irradiated $\mathrm{Fe}-10 \% \mathrm{Cr}$ [6]. In summary, although fraught with potential pitfalls and seemingly countless opportunities for getting the wrong answer, traditional diffraction-contrast analysis still has much to offer for the characterization of dislocation structures, especially for point-defect clusters such as dislocation loops [7]. 
1. G.W. Groves and A. Kelly, Philos. Mag. 6 (1961) 1527; Philos. Mag. 7 (1962) 892.

2. D.M. Maher and B.L. Eyre, Philos. Mag. 23 (1971) 409.

3. C.M. van der Walt, Philos. Mag. 24 (1971) 999; D.M. Maher and B.L. Eyre, Philos. Mag. 26

(1972) 1233; C.M. van der Walt, Philos. Mag. 26 (1972) 1237.

4. J. Bentley, PhD thesis, University of Birmingham, England (1974).

5. J. Bentley and B.A. Pint, Microsc. Microanal. 7(Suppl.2) (2001) 1246.

6. L.L. Horton et al., J. Nucl. Mater. 108/9 (1982) 222; J. Nucl. Mater. 103/4 (1981) 1085.

7. Research at the ORNL SHaRE User Facility (JB) supported by the Office of Basic Energy

Sciences, U.S. Department of Energy, under contract DE-AC05-00OR22725 with UT-Battelle, LLC.

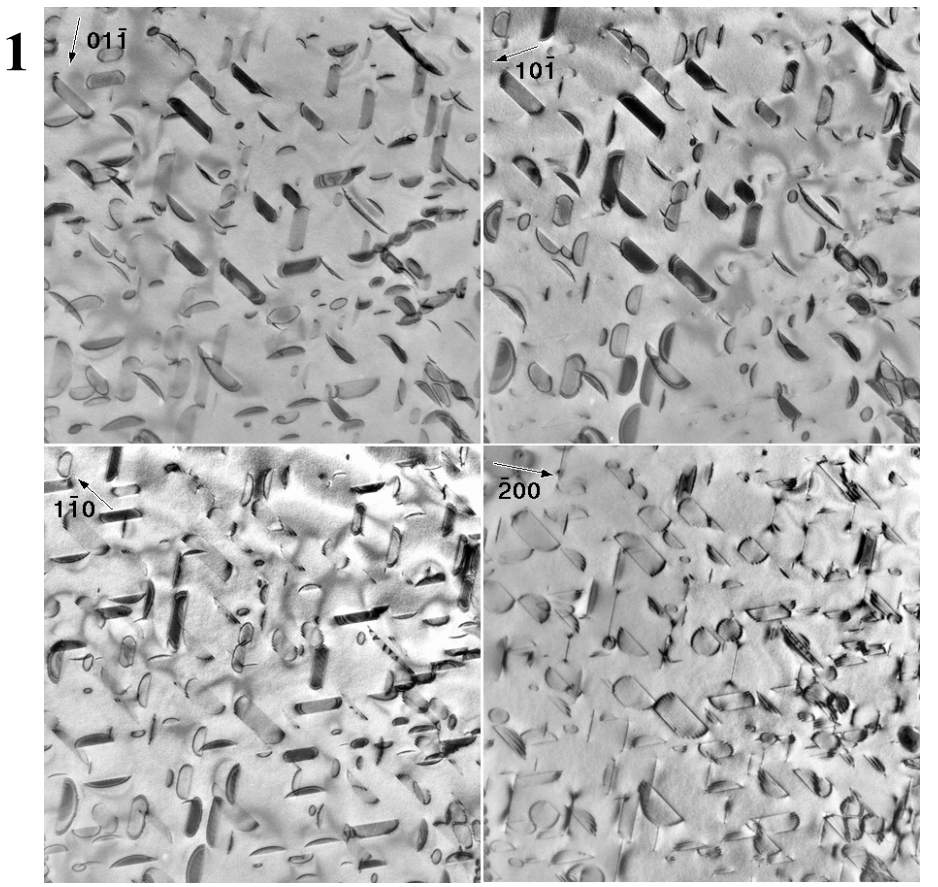

Fig. 1. Disk-shaped TiC-rich $\mathrm{Ti}(\mathrm{C}, \mathrm{O}, \mathrm{N})$ precipitates on $\{001\}$ in oxygen-doped $\mathrm{V}-4 \% \mathrm{Cr}-4 \% \mathrm{Ti}$ annealed at $950^{\circ} \mathrm{C}$ exhibiting misfit dislocation loop contrast. Pairs of $\mathbf{g . b}=0$ conditions with $\mathbf{g}= \pm 01 \overline{1}, \pm 1 \overline{1} 0, \pm 10 \overline{1}$ and 200 ( \pm g recorded but not shown) at $\mathbf{B}=$ [122], [223], [212] and [023], respectively, reveal that $\mathbf{b} \approx \mathbf{a}<001>$.

Fig. 2. Same area as Fig. 1 showing g.b $\approx \pm 2$ inside/outside contrast analyses with $\mathbf{g}= \pm \overline{1} 2 \overline{1}$, $\pm 21 \overline{1}$ and $\pm \overline{1} \overline{1} 2$ at $\mathbf{B}=$ [234] [146] and [243], respectively. The loops have interstitial character as expected from the bi-phase crystallography.

2
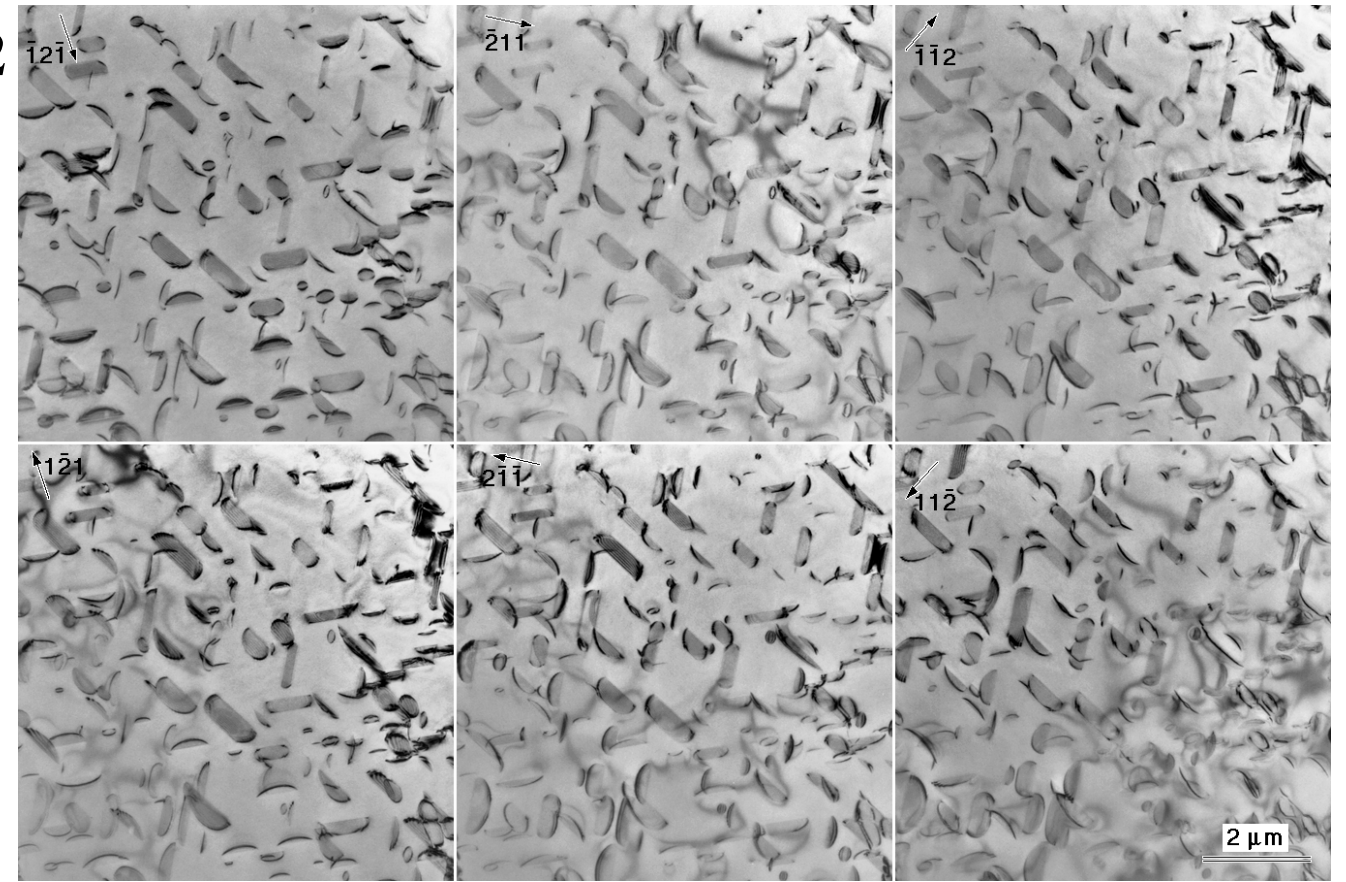